\title{
Local Wideband Spectrum Sensing Dynamic Algorithm Based on Compressive Sensing
}

\author{
Evelio Astaiza-Hoyos ${ }^{1,2}$, Pablo Jojoa ${ }^{2}$, Héctor Bermúdez $^{1}$ \\ ${ }^{1}$ GITUQ - Facultad de Ingeniería, Universidad del Quindío (UQ) \\ Carrera 15 , calle $12 \mathrm{~N}$, Armenia, Colombia \\ \{eastaiza, hfbermudez\}@uniquindio.edu.co \\ ${ }^{2}$ GNTT-Facultad de Ingeniería Electrónica y Telecomunicaciones, Universidad del Cauca (UC) \\ Calle 5, \# 4-70, Popayán, Colombia \\ \{eastaizah,pjojoa\}@unicauca.edu.co
}

\begin{abstract}
This article presents a novel algorithm to make wideband spectrum sensing in Cognitive Radio (CR) systems based on Compressive Sensing (CS), which permits to cognitive users to sense the spectrum without need for a priori knowledge of signal characteristics in the radio environment, minimizing the amount of samples to process. Simulation results demonstrate that the proposed method permits carrying out spectrum sensing efficiently, improving the performance of sensing in function of the detection probability and of the receptor's operational characteristics with respect to other algorithms proposed based on sub-Nyquist sampling.
\end{abstract}

Keywords: Compressive Sensing, Covariance Estimation Matrix, Energy detection, Sub-Nyquist sampling, Wideband spectrum sensing.

\section{INTRODUCTION}

Cognitive Radio (CR) [1] plays an important role in next-generation mobile and wireless communications systems, given that it is a technology in which cognitive devices detect automatically stimuli in their radio environment and adapt intelligently their operation parameters, guaranteeing satisfaction of the communication needs of the cognitive user (Secondary Users - SU), using efficiently and opportunistically the spectral resources not used by licensed users (Primary Users - PU) who have the right to use the spectrum.

To achieve this purpose, CR devices must have the capacity to identify with high probability locating the white spaces (WS) (sub-band occupied only by noise) available in a wideband of interest. For this, it is necessary to implement in the CR device mechanisms of wideband spectrum knowledge, like databases for spectrum access or wideband spectrum sensing. Knowledge of wideband spectrum implies, unlike that of narrowband (identifies white spaces on a band of a few $\mathrm{KHz}$, generally equivalent to a radio channel of a particular primary system), that the CR seeks to identify white spaces existing in a frequency band from tens of $\mathrm{MHz}$ to a few $\mathrm{GHz}$, where multiple radio channels of multiple primary systems generally exist, to reach higher rates of opportunistic performance, thereby, the wideband on which spectrum knowledge is sought may be divided into multiple narrowband sub-bands with the desire to know which correspond to white spaces.

Spectrum knowledge by the CR device, as mentioned, can be performed through two general mechanisms. The first, by using access databases to the spectrum [2], a mechanism that is implemented by using centralized databases that contain information of the white spaces existing in the area of influence, which are periodically updated by the primary operators, indicating the sub-bands used in the system's geographic region of influence; thus, cognitive users can consult the database and obtain information of the white spaces existing in their vicinity. This solution is perceived as effective because it provides information on the spectrum available in a given area, but not in real time; its principal disadvantage lies on the need for additional players in the CR scenario, like the database operator or operators and the infrastructure required to operate the mechanism, implying high signaling rates and involving a complex scenario and of costly implementation. The second mechanism is denominated spectrum sensing [3], [4], which requires CR devices to permanently detect activity from PU in the communications channel in the band of interest; the main spectrum sensing mechanisms discussed in literature are energy detection [5]-[10], detection based on adapted filter [6], [8], [10], [11], detection of characteristics [12]-[14], and detection based on Eigenvalues [15, 16]. In general, the spectrum sensing mechanisms mentioned are implemented by using algorithms that digitize the signal at rates equal to or above the Nyquist rate, implying in the wideband scenario processing of a great amount of samples, thus, requiring high processing capacity of the platforms that will support the sensing algorithms [17]. 
Within this context, one of the main challenges faced in CR is the efficient implementation of wideband spectrum sensing, minimizing the sampling rate required and guaranteeing high detection probability and low probabilities of miss detection and false alarm; understanding as detection probability that probability of correct detection of a white space or presence of signal from a PU. By false alarm probability, we should understand the probability associated to detecting signals from a PU when in the channel, there is actually white space and by miss detection probability, we understand that probability associated to detecting a white space when in the channel there is presence of signals from a PU. This is why in literature multiple alternatives are available to perform wideband spectrum sensing; among these, the best accepted due to its simplicity of implementation is the wideband energy detection [18], [19]; however, it requires sampling rates equal to or above the Nyquist rate and presents high sensitivity to noise. Recently, other works [20]-[22] propose wideband spectrum sensing methods based on compressive sensing, which permits reducing the amount of samples processed, but present performances lower than those obtained by [18], [19].

In recent years, based on the idea of sparse occupation of the communications channel (i.e., few frequency bands occupied and multiple bands available), many proposals have been worked based on Compressive Sensing [23], [24], which provides an efficient way of processing sparse signals or signals that can be approached adequately by sparse signals, that is, that can be approached by an expansion in terms of an adequate base, which only has some significant terms, which integrates sampling, compression, reduction of dimensionality, and optimization that would permit carrying out wideband spectrum sensing by digitizing the signal at lower rates than the Nyquist rate; the proposed techniques that allow conducting non-uniform sampling of sparse signals are: Analogue to Information Conversion (AIC) [25], [26], which is based on the idea of doing conducting nonuniform sampling of the signal at the rate of information, Modulated Wideband Conversion (MWC) [25], [27], based on generalized sampling and on the modulation and filtering of the signal to sample over multiple branches, each with different modulating functions, which permit exploiting the characteristics of the multiband signals in the frequency domain to do non-uniform sampling that permit structuring the sub-sampled signal in the frequency domain; finally, MultiCoset Sampling (MCS) [25], [28], [29], which seeks to reduce the amount of samples obtained from multiband signals, considering that the support for these signals in the frequency domain is a union of finite intervals.

Consequently, within the context described and in the search to overcome the challenge already indicated, this work's principal contribution is an algorithm based on Compressive Sensing that permits doing the wideband spectrum sensing function, minimizing the amount of samples to process and which has higher performance in terms of detection, false alarm and miss detection probabilities to that obtained in [18]-[22]; this generates a significant contribution in reducing the computational complexity required to implement $\mathrm{CR}$, allowing the cognitive user to have broader awareness of the spectrum available and, thus, be able to use a greater amount of spectral opportunities and, thereby, achieve higher rates of information transfer or satisfy those required.

In this work, to carry out the wideband spectrum sensing function in CR, a method based on Compressive Sensing and on the estimation of second-order statistics (covariance matrix) is proposed of the signal received from the samples obtained from the multiband signals taken with an Analog to Information Converter (AIC), thus, the second-order statistics of the signal present in the channel is estimated, and from the components present in the principal diagonal of the covariance matrix estimated to detect the occupation or not of the channels that conform the sensed multiband, evaluating its efficiency in function of the detection, false alarm and miss detection probabilities.

The rest of the article is organized in the following manner: section II proposes the System model; section III presents the algorithm of estimation of the covariance matrix of the multiband signal from the covariance matrix of the samples taken with the AIC denominated Random Demodulator (RD) [30]; section IV presents the performance evaluation of the proposed algorithm, contrasting the evaluation metrics against those obtained from the sequential energy detection algorithm that operates at sampling rates equal to or above the Nyquist. Finally, section $\mathrm{V}$ presents the conclusions of the conducted study.

\section{SYSTEM MODEL}

Considering a single-antenna $\mathrm{CR}$ device that operates over a multiband (licenced) with a total bandwidth of $B H z$, which is divided into non-overlapping $k$ sub-bands of equal bandwidth $b$, equivalent to $B / \mathrm{kHz}$ per channel, as shown in Fig. 1.

Assuming that the multiband signal samples are independent random variables that follow a normal distribution of zero mean and $\sigma_{s}$ variance $\left(N\left(0, \sigma_{s}\right)\right)$, a presumption that is valid for any multiband signal in which each carrier of a sub-band is modulated independently by data-streams; and that noise samples in each 
antenna are random variables normally distributed, independent, of zero mean and $\sigma_{n}$ variance $\left(N\left(0, \sigma_{n}\right)\right)$, signal received in the antenna of the CR device can be expressed as indicated in Eq.(1).

$$
\mathbf{x}_{j}(n)=\mathbf{h}_{j} \mathbf{s}_{j}(n)+\mathbf{w}_{j}(n)
$$

where $\mathbf{x}_{j}(n)$ is the $n^{-t h}$ component of the signal received by the SU in the $j^{-t h}$ sub-band with $j=1,2, . ., k, \mathbf{h}_{j}$ represents the channel response in the $j^{-t h}$ sub-band, $\mathbf{s}_{j}(n)$ is the $n^{-t h}$ component of the signal transmitted by the $j^{- \text {th }} \mathrm{PU}$ on the $j^{- \text {th }}$ sub-band and received by the SU antenna and $\mathbf{w}_{j}(n)$ is the $n^{-t h}$ noise component in the $j^{-t h}$ sub-band.

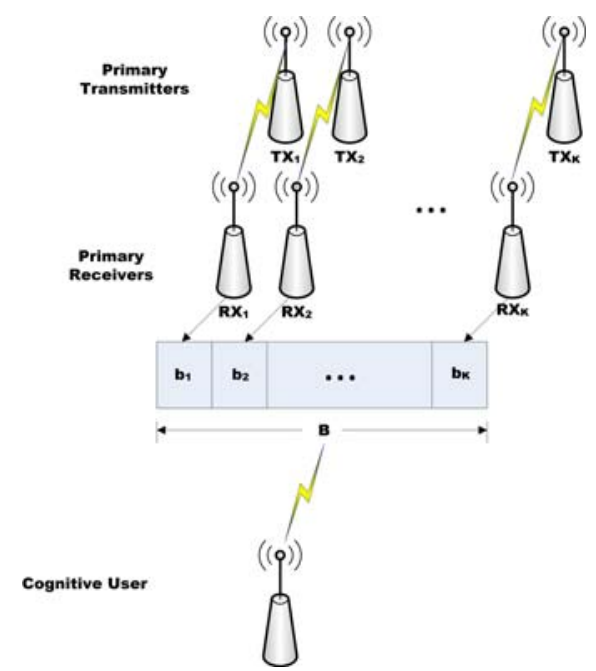

Fig. 1. Wideband spectrum sensing scenario with single antenna cognitive user. Source: [By the authors]

The spectrum sensing problem in the $j^{-t h}$ sub-band can be formulated as a statistical hypothesis testing problem in which a selection must be made between the hypothesis $H_{0, j}$ which indicates that the $j^{- \text {th }}$ sub-band is available, and hypothesis $H_{1, j}$ which indicates that the $j^{-t h}$ sub-band is occupied; the aforementioned can be expressed according to Eq.(2).

$$
\left\{\begin{array}{l}
H_{0, j}: \mathbf{x}_{j}=\mathbf{w}_{j} \\
H_{1, j}: \mathbf{x}_{j}=\mathbf{h}_{j} \mathbf{s}_{j}+\mathbf{w}_{j}
\end{array}\right.
$$

where $\mathbf{x}_{j} \in \mathfrak{R}^{p}$ is the vector of the received signal by the SU in the $j^{-t h}$ sub-band, with $p$ equal to the amount of samples taken per sub-band; $\mathbf{w}_{j} \in \mathfrak{R}^{p}$ is the vector representing the white noise components present in the $j^{-t h}$ sub-band; $\mathbf{h}_{j} \in[0,1]$ represents the channel response in the $j^{-t h}$ sub-band; finally, $\mathbf{s}_{j} \in \mathfrak{R}^{p}$ is the vector representing the signal transmitted by the $j^{- \text {th }}$ PU on the $j^{- \text {th }}$ sub-band.

To identify WS, the CR device must implement spectrum sensing by executing functions associated to each of the blocks of the diagram shown in Fig. 2. 


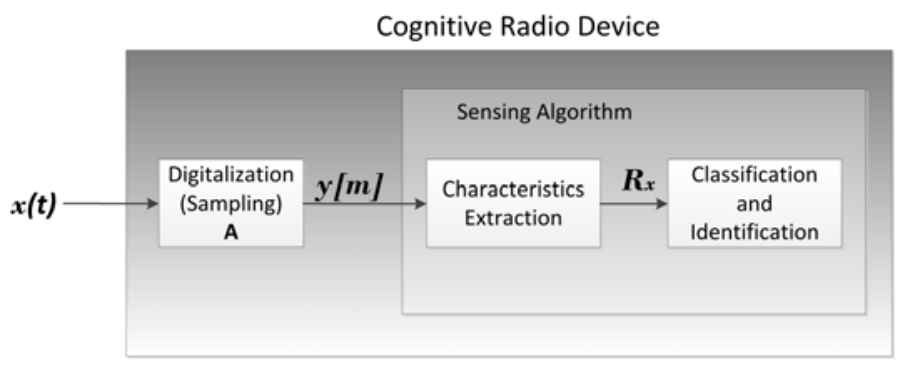

Fig. 2. Block Diagram Spectrum Sensing Function CR Device.

Source: [By the authors]

where $\mathbf{x}(t)$ represents the wideband signal in the communications channel in the licensed operator band. Initially, in the digitalization block, signal $\mathbf{x}(t)$ is sampled with the $\mathrm{RD}$, which implements sampling through the $\mathbf{A} \in \mathfrak{R}^{m \times n}$ matrix, obtaining the samples vector $\mathbf{y} \in \mathfrak{R}^{m}$, with $\mathbf{y}=\mathbf{A} \mathbf{x}$. Thereafter, the block of characteristics extraction permits obtaining the signal covariance matrix $\mathbf{R}_{x} \in \mathfrak{R}^{n \times n}$ from the samples covariance matrix $\mathbf{R}_{y} \in \Re^{m \times m}$, where $m<n$. Finally, the classification block detects WS based on the covariance matrix estimated from the signal, described in literal $\mathrm{C}$ of this section.

The following describes model of the signal and of the functions performed by the digitalization and sensing algorithm blocks.

\section{A. Model of the Signal}

Assuming that the system has time instances when the totality of the sub-bands available are not in use, and denoting by $L$ the number of channels occupied by the Primary Users active in the network, then we have $k-L$ channels available in the system that can be used by the Secondary Users of Cognitive Radio.

The order of the sparse signal in the band of the primary operator is really unknown, but given that the spectrum sensing problem only requires identifying the active carriers within the licensed bandwidth; this sparse order can be assumed as the maximum possible, which is equivalent to having $k$ operator channels occupied by Primary Users, then, the sensing signal in this case is $k$-sparse, with $k$ sparse order.

The spectrum sensing function made by the cognitive terminal is to determine occupation of the $k$ channels in the band assigned to the primary operator, where the $k-L$ channels available in the network represent transmission opportunities to the cognitive users as long as the cognitive terminals are able to detect correctly the channels available in the sensing band.

Assuming that Primary Users use a digital modulation scheme, the signal present in the licensed band is represented as shown in Eq. (3):

$$
x(t)=\sum_{n=1}^{k}\left[x_{I n}(t) \cos \left(2 \pi f_{n} t+\theta_{n}\right)-x_{Q n}(t) \operatorname{sen}\left(2 \pi f_{n} t+\theta_{n}\right)\right]
$$

Where $x_{I n}(t)$ is the in-phase component of the signal on the $n^{-t h}$ carrier of frequency $f_{n}, x_{Q n},(t)$ is the quadrature component of the signal on the $n^{- \text {th }}$ frequency carrier $f_{n}$, and $\theta_{n}$ represents the carrier signal phase. The multiband signal will appear as shown in Fig. 3.

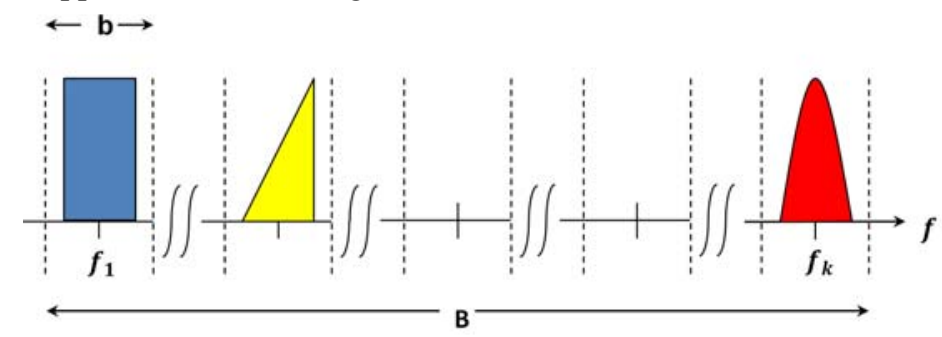

Fig. 3. Multiband Signal. Source: [By the authors] 


\section{B. SubNyquist Sampling}

The sampling of the multiband signal $x(t)$, illustrated in Eq. (1), carried out through RD, may be considered a new type of sampling system, which can be used to acquire band limited sparse signals [31].

As shown in the diagram of Fig. 4, the input signal to the random demodulator is multiplied by a high-rate pseudo-random sequence, which disperses the energy of the tones over the bandwidth total occupied by the sequence, then; anti-aliasing filtering is applied to finally sample the signal at a lower rate than the Nyquist rate. The demodulation process (multiplication by the pseudo-random sequence) guarantees that each tone present in the input signal has a different "signature" within the filter's passing band, given that the input signal to the random demodulator is only made up of some tone, it is possible to identify the tones and their amplitudes from the low-rate samples.

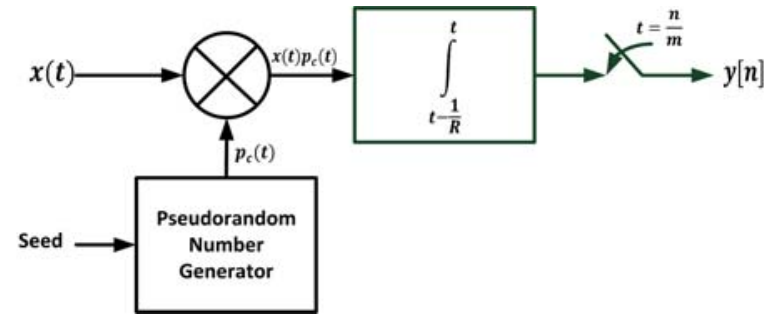

Fig. 4. Block Diagram of Random Demodulator. Source: [By the authors]

From the sub-Nyquist sampling process, we obtain samples, as illustrated by Eq. (4) [31]

$$
\mathbf{y}=\mathbf{A x}
$$

Where $\mathbf{A}$ is the sensing matrix of size $m \times n, \mathbf{y} \in \mathfrak{R}^{m}$ is the measurement vector, and $x \in \mathfrak{R}^{n}$ is the vector representing the $k$-sparse multiband signal; thereby, the inputs of $\mathbf{y}$ are the sub-Nyquist samples of $\mathbf{x}$.

\section{Spectrum sensing}

From Eq. (4), it can be noted that upon calculating the covariance matrix of the $y$ samples, we have the relation given by Eq. (5).

$$
\mathbf{R}_{\mathbf{y}}=\mathbf{A} \mathbf{R}_{\mathbf{x}} \mathbf{A}^{\mathbf{T}}
$$

with $\mathbf{R}_{x}$ being the covariance matrix of the signal present in the communications channel of size $n \times n$ and $\mathbf{R}_{y}$ is the covariance matrix of the samples taken with the RD of size $m \times m$.

Consequently, from the sample's covariance matrix it is possible to obtain the covariance matrix of the signal in the channel and with it perform the wideband spectrum sensing operation, identifying the energy present in each of the $k$ sub-bands.

The spectrum sensing function, in this order of ideas, can be conducted through identification of the values present in the principal diagonal of the estimated covariance matrix, $\mathbf{R}_{x}$, which is compiled in Eq. (6).

$$
\mathbf{R}_{x}(i, i)=\left\{\begin{array}{c}
\sigma^{2}: H_{0} \\
\sigma_{x}^{2}+\sigma^{2}: H_{1}
\end{array}\right.
$$

Where $\sigma^{2}$ represents the noise power and $\sigma_{x}^{2}$ represents the signal power, then, upon identifying if Eq. (7) is complied, signal presence may be established, which is equal to validating hypothesis $H_{1}$.

$$
\mathbf{R}_{x}(i, i)>\sigma^{2}
$$

To obtain the signal covariance matrix in the $\mathbf{R}_{x}$ channel from the covariance matrix of $\mathbf{R}_{y}$, the optimization problem must be solved, as shown in Eq. (8).

$$
\min \left\|\mathbf{R}_{x}\right\|_{1} \text { subject to } \mathbf{R}_{y}=\mathbf{A} \mathbf{R}_{\mathbf{x}} \mathbf{A}^{\mathbf{T}}
$$


The proposed solution in Eq. (8) is a modification of Orthogonal Matching Pursuit (OMP) [32], where work is not with vectors, which is why the Kronecker product is not used; rather, work is done directly in matrix form, as illustrated in the following section.

\section{Classification and Identification.}

Identification of the occupation or not of each sub-band is done in two stages: 1.) decide on the preliminary occupation or not in function of the energy present in each sub-band of the signal estimated in antenna. 2.) Decide on the final occupation of the multiband according to the occupation average associated to the preliminary decisions obtained for each sub-band.

To perform energy detection for each sub-band (stage 1), the energy of the signal received is compared to a detection threshold, thus, deciding the occupation or not of a sub-band. Thereby, the energy present in each subband can be calculated according to Eq. (9).

$$
\varepsilon_{j}(f)=\left|\mathbf{h}_{j}\right|^{2} \sum_{S b_{j}}|\hat{\mathbf{X}}[f]|^{2}
$$

Where $\varepsilon_{j}$ represents energy in the $j^{-t h}$ sub-band over a sequence of $N$ samples, $S b_{j}$ represents the $j^{-t h}$ sub-band, $\mathbf{h}_{j}$ represents the channel response in the $j^{-t h}$ sub-band, and $\hat{\mathbf{X}}[f]$ represents the signal estimated in the multiband. Then, if the energy in the $j^{-t h}$ sub-band is higher than the $T_{h_{j}}\left(\varepsilon_{j}>T_{h_{j}}\right)$ decision threshold, the decision made is $H_{1, j}$ (occupied sub-band); on the contrary, the decision is $H_{0, j}$ (free sub-band - WS).

Detection probability $P_{d_{j}}$, miss detection probability $P_{m d_{j}}$, and false alarm probability $P_{f_{j}}$, in the $j^{-t h}$ subband are defined as indicated in Eqs. (10), (11) and (12).

$$
\begin{aligned}
& P_{d_{j}}=P\left(H_{1, j} \mid H_{1, j}\right) \\
& P_{m d_{j}}=P\left(H_{0, j} \mid H_{1, j}\right)=1-P_{d_{j}} \\
& P_{f_{j}}=P\left(H_{1, j} \mid H_{0, j}\right)
\end{aligned}
$$

Understanding as detection probability that correct detection probability of occupation of a sub-band or signal presence of a PU in a sub-band (decide $H_{1, j}$ when $H_{1, j}$ is true); by probability of false alarm we understand the probability associated to detecting signal from a PU when in a sub-band there is really a white space (decide $H_{1, j}$ when $H_{0, j}$ is true), and by miss detection probability we understand that probability associated to detecting a white space when in a sub-band there is presence of signal from a PU (decide $H_{0, j}$ when $H_{1, j}$ is true).

According to the central limit theorem [33], if the number of samples is sufficiently large ( $\geq 10$ in practice), the statistics test (mean and variance) of $\varepsilon_{j}$ associated to hypotheses $H_{0, j}$ and $H_{1, j}$ are normally distributed asymptotically and given by Eqs. (13) and (14).

$$
\begin{aligned}
E\left(\varepsilon_{j}\right) & =\left\{\begin{array}{l}
2 N \sigma_{n_{j}}^{2}: H_{0, j} \\
\left(S N R_{j}+N\right) \sigma_{n_{j}}^{2}: H_{1, j}
\end{array}\right. \\
\operatorname{Var}\left(\varepsilon_{j}\right) & =\left\{\begin{array}{l}
2 N \sigma_{n_{j}}^{4}: H_{0, j} \\
2\left(2 S N R_{j}+N\right) \sigma_{n_{j}}^{4}: H_{1, j}
\end{array}\right.
\end{aligned}
$$


With $\sigma_{n_{j}}^{2}$, noise energy is denoted in the $j^{-t h}$ sub-band and $S N R_{j}$ denotes the signal to noise ratio in the $j^{-t h}$ sub-band.

Then, the detection and false alarm probabilities in the $j^{\text {th }}$ sub-band can be expressed, as indicated in Eqs. (15) and (16).

$$
\begin{gathered}
P_{d_{j}}=Q\left[\frac{T_{h_{j}}-E\left(\varepsilon_{j} \mid H_{1, j}\right)}{\sqrt[2]{\operatorname{Var}\left(\varepsilon_{j} \mid H_{1, j}\right)}}\right]=Q\left[\frac{T_{h_{j}}-\left(S N R_{j}+N\right) \sigma_{n_{j}}^{2}}{\sqrt[2]{2\left(2 S N R_{j}+N\right) \sigma_{n_{j}}^{4}}}\right] \\
P_{f_{j}}=Q\left[\frac{T_{h_{j}}-E\left(\varepsilon_{j} \mid H_{0, j}\right)}{\sqrt[2]{\operatorname{Var}\left(\varepsilon_{j} \mid H_{0, j}\right)}}\right]=Q\left[\frac{T_{h_{j}}-2 N \sigma_{n_{j}}^{2}}{\sqrt[2]{2 N \sigma_{n_{j}}^{4}}}\right]
\end{gathered}
$$

Where

$$
Q(x)=\frac{1}{\sqrt{2 \pi}} \int_{x}^{\infty} e^{\frac{-t^{2}}{2}} d t
$$

Thereby, the decision threshold $T_{h_{j}}$ for a specific value of $P_{f_{j}}$ is given by (18).

$$
T_{h_{j}}=Q^{-1}\left(P_{f_{j}}\right) \sqrt[2]{2 N \sigma_{n_{j}}^{4}}+2 N \sigma_{n_{j}}^{2}
$$

Finally, the detection probabilities $P_{d}$, miss detection probability $P_{m d}$, and false alarm probability $P_{f}$, of the multiband are calculated according to Eqs. (19), (20), and (21).

$$
\begin{gathered}
P_{d}=\frac{1}{K} \sum_{j=1}^{K}\left\{Q\left[\frac{T_{h_{j}}-\left(S N R_{j}+N\right) \sigma_{n_{j}}^{2}}{\left.\sqrt[2]{2\left(2 S N R_{j}+N\right) \sigma_{n_{j}}^{4}}\right]}\right\}\right. \\
P_{m d}=\frac{1}{K} \sum_{j=1}^{K}\left\{1-Q\left[\frac{T_{h_{j}}-\left(S N R_{j}+N\right) \sigma_{n_{j}}^{2}}{\sqrt[2]{2\left(2 S N R_{j}+N\right) \sigma_{n_{j}}^{4}}}\right]\right\} \\
P_{f}=\frac{1}{K} \sum_{j=1}^{K}\left\{Q\left[\frac{T_{h_{j}}-2 N \sigma_{n_{j}}^{2}}{\sqrt[2]{2 N \sigma_{n_{j}}^{4}}}\right]\right\}
\end{gathered}
$$

\section{Wideband SPECTRUM SENSING PRoposed Method}

The idea is to reconstruct the covariance matrix, $\mathbf{R}_{x}$, from the representation of the covariance matrix, $\mathbf{R}_{y}$, as the weighted sum of the lowest amount possible of external products of the columns of matrix $\mathbf{A}$, given that the A sensing matrix, upon complying with RIP of order $k$, guarantees that its columns permit generating the space of signal $x$. Upon estimating the covariance matrix, $\mathbf{R}_{x}$, we verify in which sub-bands defined in the multiband are there recovered components, which permits identifying the sub-bands occupied and those available.

To perform the estimate of the covariance matrix of the signal from the covariance matrix of measurements, like spectrum sensing, it is important to calculate the $K$ amount of significant components of the multiband signal that permit conducting a correct detection with probability above or equal to 0.95 ; this amount of significant components represents the amount of iterations the covariance estimation algorithm must perform. Figure 5 shows the simulation results to estimate the $K$ value for different values of $B, b$, and subsampling $n / m$ factor. 
As noted in Fig. 5, experimental results permit establishing the relation existing between the number of significant components of the multiband signal and the bandwidth total of the multi-band $B$, the bandwidth of each sub-band (channel) $b$ and the sub-sampling $n / m$ factor, as indicated in Eq. (22)

$$
K=\left(\frac{n}{m}\right) B / b
$$

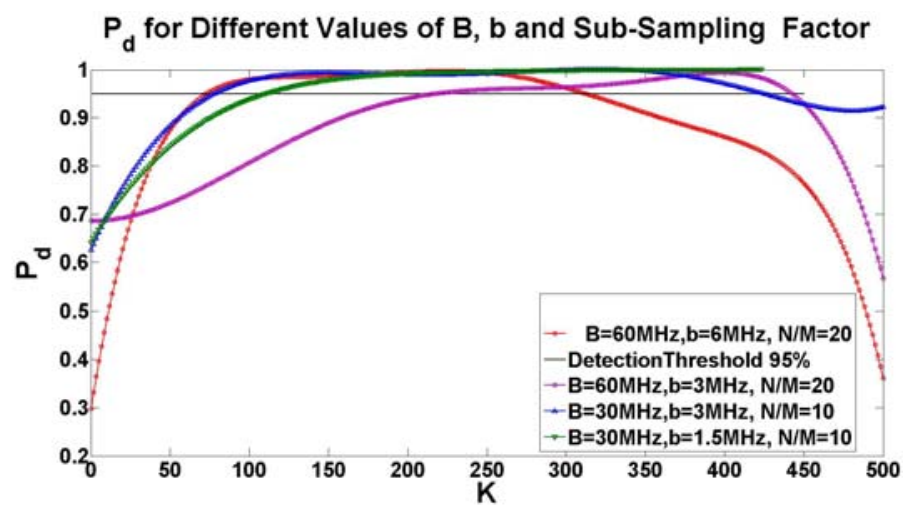

Fig. 5. Detection Probability for Different Values of B, b and Sub-Sampling Factor. Source: By the authors

\section{A Estimation of the Second-order statistics of the Signal in the channel.}

Let $\mathbf{X} \in \mathfrak{R}^{n}$ be the representation in the frequency domain of signal $\mathbf{x}$, and $\boldsymbol{\Psi} \in \mathfrak{R}^{n \times n}$ the discrete Fourier transformation matrix, such that $\mathbf{X}=F(\mathbf{x})=\Psi \mathbf{x}$ where $\mathbf{X}$ presents only $k \ll n$ significant values (inputs different from zero); upon sampling $\mathbf{X}$ with the sampling matrix $\boldsymbol{\varphi} \in \mathfrak{R}^{m \times n}$ where $k<m<n$ to obtain $\mathbf{y}=\boldsymbol{\varphi} \mathbf{X}=\boldsymbol{\varphi} \Psi \mathbf{x}=\mathbf{A x}$; if $\boldsymbol{\varphi}$ fulfills the restricted isometry property (RIP) of order $k$ [28], [29] and has low coherence with $\boldsymbol{\Psi}$, then $\mathbf{X}$ may be effectively recovered from $\mathbf{y}$. To carry out the estimation process of the signal's covariance matrix in the channel and solve the problem posed in Eq. (8), we need to use two auxiliary variables. The first of these $(i, j)$ to avoid re-selecting external products, coordinates $(i, j)$ keep the indices of the external products that can be selected. The second $\mathbf{R} \in \mathfrak{R}^{m \times n}$ to store the remainders produced upon removing the external products selected from $\mathbf{R}_{y}$. Initially, $\mathbf{R}$ is equal to $\mathbf{R}_{\mathbf{y}}$ and variable $(i, j)$ starts with all the possible combinations of indices of external products of the columns of the sensing matrix $(\mathbf{i}, \mathbf{j}) \leftarrow\{(1,1),(1,2), \ldots,(n, n)\}$; then the external product is selected that best adapts to the remainder through $\left(i_{t}, j_{t}\right) \leftarrow \underset{\left(i^{\prime}, j^{\prime}\right) \in(i, \mathbf{j})}{\arg \max } \frac{\left|\left\langle\mathbf{R}, \mathbf{P}_{i^{\prime}, j^{\prime}}\right\rangle\right|}{\left\|\mathbf{P}_{i^{\prime}, j^{\prime}}\right\|_{2}}$, excluding from the indices those corresponding to the external product selected and calculating the weights associated to each external product selected through least squares $\hat{\mathbf{u}} \leftarrow \underset{u}{\arg \min }\left\|\mathbf{R}_{\mathbf{y}}-\sum_{t^{\prime}=1}^{t} u_{t^{\prime}} \mathbf{P}_{i^{\prime}, j^{\prime}}\right\|_{2}$; then the remainder is updated, according to the external products selected and associated weights $\mathbf{R} \leftarrow \mathbf{R}_{\mathbf{y}}-\sum_{t^{\prime}=1}^{t} \hat{u}_{t^{\prime}} \mathbf{P}_{i^{\prime}, j^{\prime}}$. The process is carried out on $K$ occasions to obtain the estimated covariance matrix, $\hat{\mathbf{R}}_{x}$, in which all its inputs are zero, except in the $K$ inputs that correspond to the external products selected, inputs assigned the calculated weighted values.

In the channel covariance estimation process, we have that $\mathbf{A}=\left(\mathbf{a}_{\mathbf{1}}, \mathbf{a}_{2}, \ldots, \mathbf{a}_{\mathbf{n}}\right)$, where $\mathbf{a}_{\mathbf{i}}$ is the $i^{\text {th }}$ column of $\mathbf{A}$; thus, the external products are defined as $\mathbf{P}_{i, j}=\mathbf{a}_{\mathbf{i}} \mathbf{a}_{\mathbf{j}}{ }^{\mathbf{T}}$, permitting to represent the covariance of the samples as the weighted sum of external products, according to Eq. (23). 


$$
\mathbf{R}_{y}=\sum_{i=1}^{n} \sum_{j=1}^{n} z_{i, j} \mathbf{P}_{i, j}
$$

\section{B. Description of the Wideband Spectrum Sensing algorithm proposed.}

To implement spectrum sensing, according to Eq. (7), the algorithm illustrated in in Table I is proposed, where the algorithm input parameters are: the sensing matrix (A), the channel's samples vector (y), the multiband total bandwidth $(B)$, the bandwidth of each sub-band $(b)$, the size of the samples vector $(m)$, and the size of the signal vector $(n)$ (line 1 ); the algorithm returns the vector of sub-bands occupied and available in the multiband ( ch ) (line 2); two auxiliary variables are used, Psb to store power per sub-band of the multiband (line 3) and Pc (line 4), which stores the power from each signal component. The spectrum sensing process starts by calculating the number of sub-bands in the multiband (line 7) and the number of significant components of the multiband (line 8). Thereafter, the covariance matrix is estimated through the Covariance Estimation function (line 9) and the vector of the principal diagonal of the estimated covariance matrix is obtained (line 11), which represents the power from the signal's estimated components. Afterward, the power estimated per sub-band is calculated (line 13); finally, the presence or non-presence of signal in each sub-band is estimated (lines 14 to 17 ).

TABLE I

Dynamic Wideband Cognitive Sensing (DWBCS) algorithm

\begin{tabular}{|c|c|}
\hline & Algorithm: DWBCS \\
\hline 1 & Input: $\mathbf{A}, \mathbf{y}, m, n, B, b$ \\
\hline 2 & Output: ch \\
\hline 3 & Var: Psb \% Power per Sub-band \\
\hline 4 & Pc \% Power Signal Components \\
\hline 5 & Start \\
\hline 6 & Cont $=0$ \\
\hline 7 & $k=B / b$ \\
\hline 8 & $K=(m / n) k$ \\
\hline 9 & $\mathbf{R}_{\mathbf{x}} \leftarrow$ Co variance_Estimation. $(\mathbf{A}, \mathbf{y}, K)$ \\
\hline 10 & For $i=1$ to $n$ \\
\hline 11 & $\mathbf{P c}(i) \leftarrow \mathbf{R}_{\mathbf{x}}(i, j)$ \\
\hline 12 & For $j=1$ to $k$ \\
\hline 13 & $\operatorname{Psb}(j) \leftarrow \operatorname{sum}(\mathbf{P c}(C o n t * n / k)$ to $\mathbf{P c}(\operatorname{Cont}+1) * n / k))$ \\
\hline 14 & If $\operatorname{Psb}(j) \geq 0$ then \\
\hline 15 & $\operatorname{ch}(j) \leftarrow 1$ \\
\hline 16 & Else \\
\hline 17 & $\operatorname{ch}(j) \leftarrow 0$ \\
\hline 18 & End if \\
\hline 19 & Cont $=$ Cont +1 \\
\hline 20 & End For \\
\hline 21 & End For \\
\hline 22 & Return ch \\
\hline
\end{tabular}

\section{PERFORMANCE EVALUATION}

This section analyzes the performance of the algorithm proposed in a scenario presenting a multiband signal composed of six channels (3.3 MHz each), which randomly present occupation. The objective is to evaluate the conditions under which the algorithm presents adequate spectrum sensing performance, compared to the performance presented by the energy sequential detection algorithm, which functions at sampling rates equal to or above the Nyquist rate. 


\section{A Scenario and Simulation parameters}

In implementing the simulation of the wideband spectrum-sensing algorithm proposed, a multiband signal is generated in a range between 0 and $20 \mathrm{MHz}$ in which six sub-frequency bands are defined; each primary user transmits a rate of $2 \mathrm{Msps}$, using 16 QAM modulators with a roll-off factor of 0.5 . The simulation parameters are shown in Table II.

TABLE II

Simulation Parameters

\begin{tabular}{|c|c|}
\hline Parameter & Value \\
\hline \multicolumn{2}{|c|}{ Multiband Signal Generation } \\
\hline Multiband Signal Bandwidth, $B W$ & $20 \mathrm{MHz}$ \\
\hline Bandwidth per Channel, $B$ & $3.3 \mathrm{MHz}$ \\
\hline Signal Modulation in Channel & 16 QAM \\
\hline Symbol rate, $R_{s}$ & 2 Msps \\
\hline Transmission Filter Roll Off Factor, $\rho$ & 0.5 \\
\hline Sampling Nyquist Sampling Rate, $f_{s}$ & $40 \mathrm{MHz}$ \\
\hline $\begin{array}{l}\text { Number of Multiband Signal Samples } \\
\text { (Nyquist), } n\end{array}$ & 2000 \\
\hline Carrier Frequencies, $f_{c}$ & $\begin{array}{c}3 \mathrm{MHz}, \\
6 \mathrm{MHz}, \\
9 \mathrm{MHz}, \\
12 \mathrm{MHz}, \\
15 \mathrm{MHz} \text { and } \\
18 \mathrm{MHz}\end{array}$ \\
\hline \multicolumn{2}{|c|}{ Sub-Nyquist Sampler (Random Demodulator) } \\
\hline Number of Input Signal Samples, $n$ & 2000 \\
\hline Number of Output Signal Samples, $m$ & 100 \\
\hline \multicolumn{2}{|c|}{ WBCS sensing algorithm } \\
\hline Number of Iterations, $K$ & 200 \\
\hline
\end{tabular}

\section{B. Metrics, Characterization and Comparison}

To evaluate the performance of the spectrum sensing algorithm proposed, the detection probability in function of the signal to noise ratio of the multiband generated and the Receptor Operational Characteristic (ROC) curves are used as metrics, compared to the metrics obtained from the sequential energy detection algorithm [18], [19], and the algorithms based on Compressive Sensing proposed by Haque [20], Sun [21], and Wang [22].

Figure 6 shows the performance of the algorithm proposed in function of the detection probability against the performance of the energy detector [18], [19] and against the performance of the algorithms based on Compressive Sensing proposed in [20]-[22]. Note, from the figure, that performance of the algorithm proposed improves significantly the performance of the other algorithms, particularly under conditions of low SNR (SNR below $5 \mathrm{~dB}$ ), given that the detection probability is higher than that obtained by the other algorithms used for comparison.

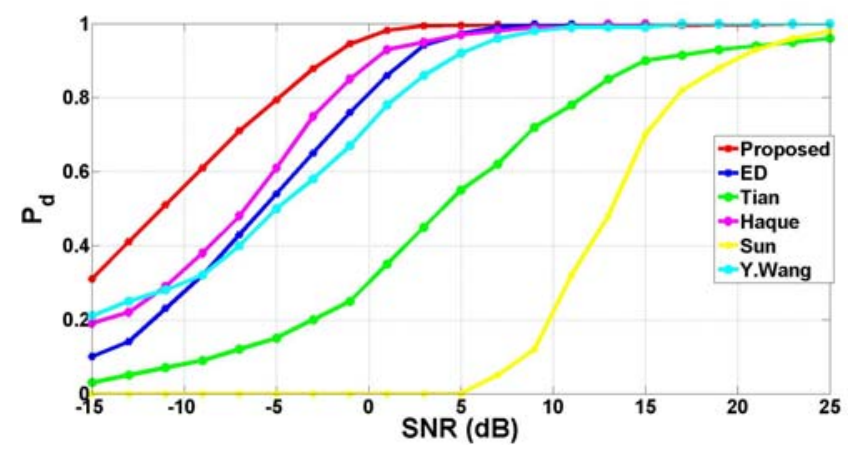

Fig. 6. Detection Probability for Different SNR Values. Source: By the authors

Figure7 reveals that the best performance in terms of ROC curves corresponds to the algorithm proposed; this is because the area below the curve of the algorithm proposed is the biggest, indicating the capacity of the algorithm proposed to identify correctly the WS. As also noted in Fig. 7, the algorithm with the worst 
performance is that proposed by Sun [21], given that the ROC curve indicates a probability of 0.5 of correctly detecting the WS. Considering that the results illustrated in Fig. 8 correspond to the ROC curves of the five algorithms contrasted to an SNR of $1 \mathrm{~dB}$, it is further evidence that the algorithm proposed improves significantly the performance of the other algorithms under low SNR conditions.

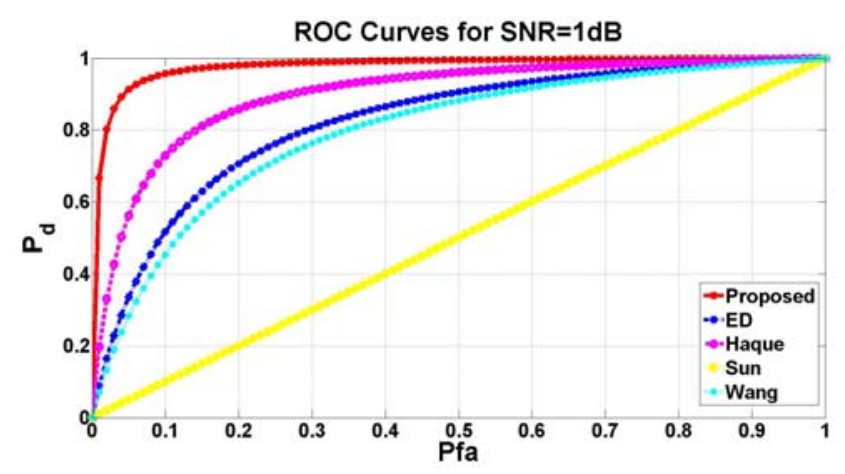

Fig. 7. ROC curves for $\mathrm{SNR}=1 \mathrm{~dB}$.

Source: by the authors

\section{Computational complexity analysis}

The computational complexity of the algorithm proposed may be analyzed in three stages. The first stage corresponds to the sub-sampling performed with the $\mathrm{RD}$, which require an amount of $\mathrm{Km}$ scalar multiplications [17]. The second stage, which calculates the covariance matrix of the samples and which is obtained from $\mathrm{m}^{2}$ scalar multiplications, then, the signal's covariance matrix is reconstructed. In the algorithm described in section $3 \mathrm{~A}$ it is possible to identify that the projection operation is limited by $O\left(m n^{2}\right)$, given that $m<n$. The selection operation of the best external product has complexity $O\left(n^{2}\right)$, given that $n^{2}$ external products exist. The weight selection operation has complexity $O\left(m^{2}\right)$, given that the highest amount of operations is represented by the product of size matrices $(m \times m)$ of the internal product associated to the least squares optimization problem. Upon finalizing this stage, we find the remainder upgrade operation, which presents complexity $O\left(K^{2}\right)$. In conclusion, given that the maximum complexity of the operations carried out in this stage is that associated to the projection operation, then the complexity of the complete stage is $O\left(m n^{2}\right)$. The third and last stage is that corresponding to the consolidation of sensing, where, as illustrated in Fig. 5, the signal covariance estimation operation (stage 2) presents complexity $O\left(m n^{2}\right)$; thereafter, lines 10 to 22 of Fig. 5 have complexity $7 k+3 n+1$ and given that $n k$ complexity may be defined as $O(n)$ with which it is defined as complexity of the third stage $O\left(m n^{2}\right)$.

In synthesis, the computational complexity of the three stages that make up the method proposed will be the maximum complexity of the three previous stages $O\left(m n^{2}\right)=\max \left[K m, O\left(m n^{2}\right), O\left(m n^{2}\right)\right]$.

\section{CONCLUSIONS}

This article presents a wideband spectrum energy sensing dynamic algorithm based on sub-Nyquist sampling and on recovery of the second-order statistics of the multiband signal in the channel, which requires no prior knowledge of the signal characteristics present in the radio environment, demonstrating through the proposed algorithm that it is possible to perform the function of wideband spectrum sensing using an amount of samples 20 times smaller than those obtained at the Nyquist rate, achieving higher performance under conditions of low SNR at performances obtained by algorithms, like those proposed in [18], [19], which operate at the Nyquist rate [20]-[22] based on Compressive Sensing and, consequently, operate at rates lower than the Nyquist rate. 


\section{REFERENCES}

[1] Mitola, J. Cognitive radio: An integrated agent architecture for software defined radio. Doctor of Technology, Royal Inst. Technol. (KTH), Stockholm, Sweden, 2000.

[2] ET Docket No. 04-186, DA 11-131 FCC, Second Memorandum Opinion and Order. Jan. 2011 ET Docket No. 04-186, DA 11-131.

[3] Tian, Z. and Giannakis, G. B. A Wavelet Approach to Wideband Spectrum Sensing for Cognitive Radios. IEEE First International Conference On Cognitive Radio Oriented Wireless Networks and Communications, pp. 1-5, 2006.

[4] Quan, Z., Cui, S., Sayed, A. H. and Poor, H. V. Wideband Spectrum Sensing in Cognitive Radio Networks. IEEE Transactions on Signal Processing, pp. 901-906, 2008.

[5] Sonnenschein, A. and Fishman, P. M. Radiometric detection of spreadspectrum signals in noise of uncertainty power. IEEE Trans. Aerosp. Electron. Syst., vol. 28, no. 3, pp. 654-660, Jul. 1992.

[6] Sahai, A. and Cabric, D. Spectrum sensing: Fundamental limits and practical challenges. A tutorial in IEEE Int. Symp. New Frontiers DySPAN, Baltimore, MD, Nov. 2005.

[7] Tandra, R. and Sahai, A. Fundamental limits on detection in low SNR under noise uncertainty. in Proc. WirelessCom, Maui, HI, Jun. 2005, pp. 464-469.

[8] Kay, S. M. Fundamentals of Statistical Signal Processing: Detection Theory, vol. 2. Englewood Cliffs, NJ: Prentice-Hall, 1998.

[9] Urkowitz, H. Energy detection of unknown deterministic signals. Proc. IEEE, vol. 55, no. 4, pp. 523-531, Apr. 1967.

[10] Cabric, D., Tkachenko, A.,and Brodersen, R.W. Spectrum sensing measurements of pilot, energy, and collaborative detection. in Proc. MILCOM, Oct. 2006, pp. 1-7.

[11] Chen, H.-S., Gao, W. and Daut, D. G. Signature based spectrum sensing algorithms for IEEE 802.22 WRAN. in Proc. IEEE ICC, Jun. 2007, pp. 6487-6492.

[12] Gardner, W. A. Exploitation of spectral redundancy in cyclostationary signals. IEEE Signal Process. Mag., vol. 8, no. 2, pp. 14-36, Apr. 1991.

[13] Gardner, W. A., Brown, W. A. and Chen, C.-K. Spectral correlation of modulated signals-Part II: Digital modulation. IEEE Trans. Commun., vol. COM-35, no. 6, pp. 595-601, Jun. 1987.

[14] Han, N., Shon, S. H., Joo, J. O. and Kim, J. M. Spectral correlation based signal detection method for spectrum sensing in IEEE 802.22 WRAN systems. in Proc. Int. Conf. Advanced Commun. Technol., Phoenix Park, Korea, Feb. 2006, pp. 1765-1770.

[15] Chatzinotas, S., Sharma, S.K. and Ottersten, B. Asymptotic analysis of eigenvalue-based blind spectrum sensing techniques. in Proc. IEEE ICASSP, May 2013, pp. 4464-4468.

[16] Sharma, S. K., Chatzinotas, S. and Ottersten, B. Eigenvalue based sensing and SNR estimation for cognitive radio in presence of noise correlation. IEEE Trans. Veh. Technol., vol. 62, no. 8, pp. 1-14, Sep. 2013.

[17] Mishali, M., Eldar, Y. C., Dounaevsky, O. and Shoshan, E. Xampling: Analog to Digital at Sub-Nyquist Rates, IET Journals \& Magazines, vol. 5, No. 1, pp. $8-20,2011$.

[18] Olabiyi, O. and Annamalai, A. "Extending the Capability of Energy Detector for Sensing of Heterogeneous Wideband Spectrum", IEEE Consumer Communications and Networking Conference (CCNC), p.p 454 - 458,2012.

[19] Olabiyi, O. and Annamalai, A. Parallel multi-channel detection: A practical solution to energy detection of heterogeneous wideband spectrum. IEEE Sarnoff Symposium (SARNOFF), p.p 1 - 5,2012.

[20] Haque, T., Yazicigil, R.T., Pan, K. J., Wright, J. and Kinget, P. R. Theory and Design of a Quadrature Analog-to-Information Converter for Energy-Efficient Wideband Spectrum Sensing. IEEE Transactions On Circuits And Systems, Vol. 62, No. 2, pp. 527 - 535, February 2015.

[21] Sun, W., Huang, Z., Wang, F. and Wang, X. Compressive wideband spectrum sensing based on single channel, IEEE Electronics Letters, Vol. 51 No. 9 pp. 693-695, April 2015.

[22] Wang, Y., Guo, C., Sun, X. and Feng, C. Time-Efficient Wideband Spectrum Sensing based on Compressive Sampling. IEEE 81 Vehicular Technology Conference, pp. 1-5, 2015.

[23] Candès, E.J., Tao, J. T. and Romberg, J. Robust uncertainty principles: exact signal reconstruction from highly incomplete frequency information, IEEE Trans. Inform. Theory 52 (2006), 489-509.

[24] Baraniuk, R. Compressive sensing, IEEE Signal Processing Magazine, pp. 118-121, July 2007.

[25] Hongjian, S., Nallanathan, A., Wang, C. X. and Chen, Y. Wideband spectrum sensing for cognitive radio networks: A survey. IEEE Wireless Commun., vol. 20, no. 2, pp. 74-81, Apr. 2013.

[26] Laska, J., et al. Theory and implementation of an analog-to-information converter using random demodulation. in Proc. IEEE Int. Symp. Circuits Syst., New Orleans, LA, USA, pp. 1959-1962, May 2007.

[27] Mishali, M. and Eldar, Y.C. From theory to practice: Sub-Nyquist sampling of sparse wideband analog signals. IEEE J. Sel. Topics Signal Process., vol. 4, no. 2, pp. 375-391, Apr. 2010.

[28] Mishali, M. and Eldar, Y. C. Blind multiband signal reconstruction: Compressed sensing for analog signals. IEEE Trans. Signal Process., vol. 57, no. 3, pp. 993-1009, Mar. 2009.

[29] Yen, C.-P., Tsai, Y. and Wang, X. Wideband spectrum sensing based on sub-Nyquist sampling. IEEE Trans. Signal Process., vol. 61, no. 12, pp. 3028-3040, Jun. 2013.

[30] Tropp, J. A., Laska, J. N., Duarte, Romberg, J. K. and Baraniuk, R. G. Beyond Nyquist: Efficient sampling of sparse bandlimited signals. IEEE Trans. Information Theory, vol. 56, no.1, pp. 520-544, Jan. 2010.

[31] E. Astaiza Hoyos, P.E. Jojoa Gómez, H.F. Bermúdez Orozco, Compressive sensing: a methodological approach to an efficient signal processing, Revista DYNA, pp. 203 - 210, Aug. 2015.

[32] Tropp, J. and Gilbert, A. Signal recovery from random measurements via orthogonal matching pursuit. IEEE Trans. Inform. Theory, vol. 53, no. 12, pp. 4655-4666, DEq. 2007.

[33] B. V. Gendenko and A. N. Kolmogorov, "Limit Distributions for Sums of Independent Random Variables", Reading, MA: AddisonWesley, 1954. 


\section{AUTHOR PROFILE}

E. Astaiza-Hoyos, Electronics Engineer from Universidad of Cauca (1998). Masters in Engineering, area of Telecommunications, Universidad of Cauca (2008). Currently PhD candidate sciences of Electronics. Associate Professor at Universidad of Quindío, program of Electronic Engineering, Researcher with the Telecommunications Research group (GITUQ) at Universidad of Quindío. Areas of interest: wireless comunications, spectrum sensing.

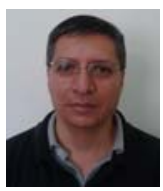

P. E. Jojoa-Gómez, Electronics Engineer from Universidad of Cauca (1993). Masters in Engineering, area of Electronic Systems, Universidad de Sao Paulo, Brazil (1999). PhD in Engineering, Area of Electronic Systems, Universidad de Sao Pablo, Brazil (2003). Coordinator of the research group on New Technologies in Telecommunications. Areas of interest: digital processing of signals and adaptive filtering.

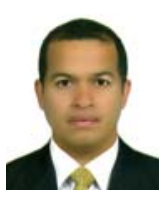

H. F. Bermúdez-Orozco, Electronics Engineer from Universidad of Cauca (2000). Masters in Electronics and Telecommunications, Universidad of Cauca (2010). Current PhD student in Telematic Engineering. Associate Professor at Universidad of Quindío, program of Electronic Engineering, Coordinator of the Telecommunications Research group (GITUQ) at Universidad of Quindío. Areas of interest: wireless comunications, radiant systems and propagation, modeling of traffic of telematic services. 